\title{
Cholestatic Jaundice in Infants - An Experience in Tertiary Care Hospital
}

\author{
FR CHOWDHURY ${ }^{\mathrm{a}}$, K CHOWDHURY ${ }^{\mathrm{b}}$, ASMB KARIM $^{\mathrm{c}}$
}

\begin{abstract}
Summary:
Background: Neonatal cholestasis is defined as prolonged elevation of serum levels of conjugated bilirubin beyond the first 14 days of life. Cholestasis in a newborn can be due to infectious, genetic, metabolic, or undefined abnormalities giving rise to mechanical obstruction of bile flow or to functional impairment of hepatic excretory function and bile secretion. Early detection and timely accurate diagnosis are important for successful treatment and a favorable prognosis.
\end{abstract}

Objective: The present study has been designed to determine the etiology of cholestatic jaundice in infants along with their clinical profile.

Methodology: This cross-sectional study was conducted from August 2010 through January 2011 in the Paediatric Gastroenterology \& Nutrition Department, Bangabandhu Sheikh Mujib Medical University (BSMMU), Dhaka. For the study purpose 40 consecutive cases of cholestatic jaundice were included who fulfilled the inclusion criteria.

Result: Biliary atresia was the commonest (42.5\%) cause of cholestatic jaundice followed by neonatal hepatitis/ idiopathic neonatal hepatitis. Other causes of cholestatic

Introduction:

Neonatal cholestasis is defined as prolonged elevation of serum levels of conjugated

bilirubin beyond the first 14 days of life ${ }^{1}$. Conjugated hyperbilirubinemia is defined by a serum conjugated bilirubin concentration of greater than $1 \mathrm{mg} / \mathrm{dl}(17.1 \mathrm{~mol} /$ l) if the total bilirubin is $5 \mathrm{mg} / \mathrm{dl}(85.5 \mathrm{mmol} / \mathrm{l})$ or more than $20 \%$. It is an abnormal finding and requires additional evaluation if it persist beyond 2 weeks of life ${ }^{2}$.

a. Dr. Farzana Rahman Chowdhury, Registrar, Shahabuddin Medical College, Gulshan, Dhaka.

b. Dr. Kona Chowdhury, Assistant Professor, Department of Paediatrics, Ad-din Women's Medical College and Hospital.

c. Prof. ASM Bazlul Karim, Professor of Paediatric Nutrition and Gastgroenterology, Bangabandhu Sheikh Mujib Medical University, Dhaka.

Address of Correspondence: Dr. Farzana Rahman Chowdhury, Registrar, Shahabuddin Medical College, Gulshan, Dhaka. Flat \# A2, DOMINNO Altura, 342, Segunbagicha, Dhaka-1000, Tel: 01715786329, E-mail: shomi9@yahoo.com

Received: 22 February, 2013

Accepted: 28 November, 2013 jaundice were choledochal cyst and hypothyroidism. Most of the infants were term and of normal birth weight in cases of biliary atresia (BA) but in NH/INH group significant number of infants were preterm and of low birth weight. Mean age at onset of jaundice was $10.1 \pm 4.18$ days, and mean age at presentation was $113.7 \pm 15.38$ days. In cases of BA Jaundice, intermittent / persistent pale stool, dark urine was found in all cases and hepatomegaly and splenomegaly were found in $88.2 \%$ and $64.8 \%$ of cases respectively. Ultrasonographically in most of the cases of $B A$ gallbladder was found either small in size or absent or bile ducts were not visualized. In cases of NH/INH visualization of normal gallbladder while fasting and contraction was observed after meal. Histologically typical features $B A$ were found in 12 out of 17 cases of $B A$ and features of early biliary cirrhosis in 4 infants and 10 patients showed features of INH.

Conclusion: Biliary atresia was found to be the commonest cause of neonatal cholestasis in the present study.

Key Words: Neonatal cholestasis, Biliary atresia, Neonatal jaundice.

(J Banagladesh Coll Phys Surg 2014; 32: 9-15)

An infant with cholestatic jaundice usually presents with prolonged jaundice, pale stool and dark urine. Acholic stool, a cardinal feature of cholestasis should be promptly evaluated.

Other clinical features depend on etiology of cholestasis. About $70 \%$ cases of neonatal cholestasis are due to biliary atresia \& Idiopathic neonatal hepatitis (INH) ${ }^{3}$. Idiopathic neonatal hepatitis occurs more commonly in males, especially those born prematurely or with low birth weight; there is a familial incidence of approximately $10 \%$ to $20 \%{ }^{4}$. In contrast, biliary atresia is more common in females of term and of normal birth weight and familial cases are rare. Affected children present with firm hepatomegaly and splenomegaly. Congenital malformations, including cardiac anomalies, polysplenia, intestinal malrotation and situs inversus, may be found in almost a third of infants with biliary atresia ${ }^{5}$.

Initially, the symptoms of BA are indistinguishable from neonatal jaundice, due to other causes. Symptoms are usually evident between one and six weeks after birth ${ }^{6}$. 
Prolonged jaundice that is resistant to phototherapy and/ or exchange transfusion should prompt a search for secondary causes. By this time, liver enzymes are generally measured, and these tend to be grossly deranged. Ultrasound investigation or other forms of imaging can confirm the diagnosis. Further testing includes radioactive scans of the liver and a liver biopsy $^{7}$.

The early detection of biliary atresia is one of the major challenges facing pediatrician when evaluating the jaundice in infant. Early recognition of liver disease greatly facilitates the care and outcome of infants. A key component of the work-up is measurement of serum conjugated bilirubin levels after 2 weeks which if elevated should prompt the clinician to initiate a workup to determine the cause of neonatal cholestasis ${ }^{8}$. In general, if patient is developing progressive jaundice soon after birth and is still jaundiced at 2 weeks of life, or develops jaundice within 3 months of life, a work up for neonatal cholestasis should begin ${ }^{9}$. The success rate for establishing good bile flow after the Kasai operation is much higher (90\%) if performed before 8 wks of life ${ }^{10}$.

Nowadays, development of sophisticated diagnostic modalities and methods makes the diagnosis possible in early stages and the underlying cause could be easily discerned. In spite of this, unfortunately there are limited data about the disease among Bangladeshi infants.

As the outcome of biliary atresia depends on early recognition and timely surgery, so the study was undertaken in infants having jaundice developed after 2 weeks of life to determine the age of onset and to document the common clinical presentation.

\section{Methodology}

\section{Study site \& duration:}

This hospital based cross sectional descriptive study was conducted at the Paediatric Gastroenterology Department, Bangabandhu Sheikh Mujib Medical University (BSMMU), Dhaka from August 2010 to December 2011.

\section{Study population:}

Admitted patient of the department were the study population and those who gave consent were enrolled.

Infants below 12 months of age are included with all of the following criteria:
1. Jaundice developed before 3 months of age and persisted for at least 2 weeks.

2. Intermitted or persistent pale colored stool.

3. Passage of dark urine

4. Conjugated bilirubin concentration more than $20 \%$ of total bilirubin or $>2 \mathrm{mg} / \mathrm{dl}$

The following babies are excluded from this study:

- Very sick infants with features of liver failure.

- Parents not willing to participate in the study.

- Patient of hepatocellular carcinoma \& hemolytic jaundice.

After enrolment patients underwent the following routine investigations:

Serum bilirubin, fractionated serum bilirubin, liver function tests like alanine aminotransferase (ALT), alkaline phosphatase, gamma glutamyl transferase and prothrombin time. Complete blood count, Blood glucose, reducing substance in urine, urine for routine microscopic examination and culture sensitivity, bacterial culture of blood, ultrasonography of liver and biliary system.

Investigations to establish a specific diagnosis: Endocrine studies (FT4, TSH ), TORCH screening, VDRL, HBsAg, serum alpha 1 antitrypsin level, hepatobiliary scintigraphy (HIDA scan), percutaneous liver biopsy were done.

Data collection method: Data were collected using a preformed data collection sheet (questionnaire). Statistical analysis was done using the statistical package for social sciences (SPSS version 17.0 for Windows, SPSS Inc. Chicago, IL). All the values were expressed as Mean $\pm \mathrm{SD}$; Students unpaired t test were applied as statistical tools. $\mathrm{p}$ value of $<0.05$ was considered as significance.

\section{Results:}

In this study a total of 40 children were studied. Biliary atresia was found to be the commonest, 17(42.5\%), cause of neonatal cholestasis followed by idiopathic neonatal hepatitis, 10 (25\%) and neonatal hepatitis, 08 (20\%). Among the neonatal hepatitis, cytomegalovirus, rubella virus and herpes simplex virus were found in 4, 2 and 2 cases respectively as identifiable causes. (Table I) 
In this study the mean age at admission of biliary atresia was $113.7 \pm 15.38$ days and that of neonatal hepatitis $105.05 \pm 16.81$ days. The overall mean age at admission of cholestatic cases was $111.9 \pm 21.14$ days. (Table II)

In this study twenty five children were male and fifteen female. Biliary atresia was present in $70.6 \%$ male $\&$ in 29.4\% female childrens and INH was present in 55.5\% male \& 44.4\% female children. (Table III)

The mean age at onset of jaundice was $10.1 \pm 4.18$ days and mean age at admission was $113.7 \pm 15.38$ days in case of biliary atresia. The mean age at onset of jaundice was $12.4 \pm 4.7$ days and mean age at admission was $105.05 \pm 16.81$ days in case of $\mathrm{NH}$ or INH. Thus an overall delay in seeking treatment was 103.6 days in biliary atresia and 92.65 days in NH \& INH cases. (Table IV)

Among the studied patient 14 (82.3\%) patients were term and 03 (17\%) were preterm in biliary atresia case but 13 (72.2\%) were term and 05 (27.8\%) preterm in $\mathrm{NH} / \mathrm{INH}$. Most of the patients in biliary atresia cases were term infants. In this study jaundice and dark urine were found in all cases of both biliary atresia and neonatal hepatitis or idiopathic neonatal hepatitis cases. Persistent acholic stool was an important finding of biliary atresia 15 (88.2\%) cases and intermittent acholic stool was a significant finding of $\mathrm{NH} / \mathrm{INH}, 14$ (77.7\%) cases. In biliary atresia 15 (88.2\%) patients were found to have hepatomegaly and the liver was firm to hard in consistency. Fourteen (77.7\%) patients with NH / INH were found to have hepatomegaly. Splenomegaly was commoner in $\mathrm{NH} / \mathrm{INH}$ case than in biliary atresia. Ascites was seen in one patient with biliary atresia and in three cases of NH / INH. None of the patients showed eye findings like cataract, posterior embryotoxon, cherry red spot or chorioretinitis. (Table V)

In biliary atresia the mean serum total bilirubin was $12.4 \pm 2.55 \mathrm{mg} / \mathrm{dl}$ and that in NH/INH was $14.7 \pm 4.64 \mathrm{mg} /$ $\mathrm{dl}$ and serum ALT wass 219.7 $\pm 102.5 \mathrm{U} / \mathrm{L}$ and $423 \pm 92.6$ $\mathrm{U} / \mathrm{L}$ in biliary atresia and NH/INH cases respectively which was not statistically significant. The mean serum alkaline phosphatase was $1048.8 \pm 162.8 \mathrm{U} / \mathrm{L}$ in biliary atresia and $629.6 \pm 160.8$ in $\mathrm{NH} / \mathrm{INH}$ cases. Gammaglutamyl transpeptidase, prothrombin time or serum albumin level showed no significant difference between biliary atresia and NH/INH groups. (Table VI) USG was done in all infants with cholestatic jaundice. In biliary atresia gall bladder was found either small in size or absent or bile channels were not visualized and no contraction of gall bladder was seen even after meals. On the contrary, in neonatal hepatitis, gall bladder was normally visualized with biliary channels and contraction of gallbladder was seen after meals. (Table VII).

HIDA scan was done in selected patients i.e. in fourteen infants. Nine patients showed delayed uptake but normal excretion, which is consistent with $\mathrm{NH}$, Where as five infants showed normal uptake of the isotope but absent excretion into the biliary channels and intestine, which is consistent with biliary atresia.

Liver biopsy was done in twenty six infants. Typical features of biliary atresia were found in twelve patients. Ten patients showed features of idiopathic neonatal hepatitis and four patients had features of early biliary cirrhosis. Liver biopsy could not be done in other cases due to prolonged prothrombin time, huge ascites or lack of parental consent. (Table VIII)

Table-I

Etiology of cholestasis in studied patients $(n=40)$

\begin{tabular}{lcc} 
Etiology & Number & Percentage \\
\hline Biliary atresia & 17 & 42.5 \\
Neonatal hepatitis: & 08 & 20 \\
$\quad$ Cytomegalovirus(CMV) & 04 & \\
$\quad$ Rubella Virus & 02 & \\
$\quad$ Herpes simplex virus & 02 & \\
Idiopathic neonatal hepatitis & 10 & 25 \\
Miscellaneous: & & 12.5 \\
$\quad$ Choledochal cyst & 3 & \\
$\quad$ Hypothyroidism with CMV & 2 & \\
\hline Total & 40 & 100 \\
\hline
\end{tabular}

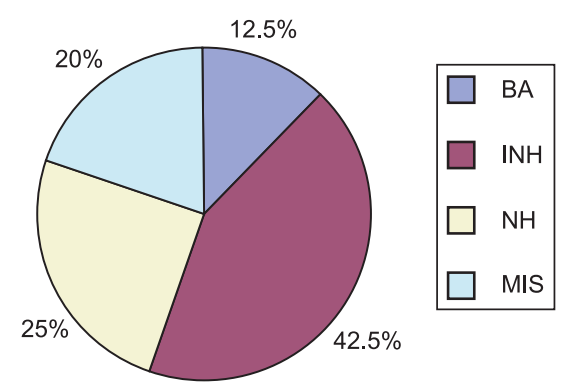

Fig.-1: Etiology of cholestasis in studied patients $(n=40)$ 
perilobular edema and fibrosis, with the intact basic hepatic lobular architechture . In biopsy, features of biliary atresia were found in 12 cases and features of early biliary cirrhosis in 4 infants. Ten patients showed features of idiopathic neonatal hepatitis. Karim and Kamal ${ }^{11}$ reported biliary atresia in 6 of 19 patients and biliary cirrhosis in four infants. They found 8 patients with idiopathic neonatal hepatitis. These findings are consistent with the present study. Though this is the main differentiating diagnostic procedure, its use is in community is limited due to lack of expertise and facilities in the wider community.

\section{Conclusion:}

Biliary atresia was found to be the commonest cause of cholestasis in this study. Most of the children presented late though appearance of jaundice was before two weeks of life. Acholic stool and USG finding before and after food appears to be differentiating and may be used by the primary care giver in identifying the problem and early referral.

\section{References:}

1. A-Kader HH, Balisteri WF. Cholestasis. In: Kliegman RM, Behrman RE, Jenson HB, Stanton BF editors. Nelson Textbook of Pediatrics. $18^{\text {th }}$ ed. New Delhi: Elsevier; 2008. p. 1668.

2. Moyer V, Frees DK, Whitington PF, Olson AD, Brewer F, Colletti RB, et al. Guidelines for evaluation of cholestatic jaundice in infants: Recommendation of the North American Society for Pediatric Gastroenterology, Hepatology and Nutrition. J Pediatr Gastroenterol and Nutr.2004;39:115-128.

3. Danks DM, Campbell PE, Jack I, et al. Studies of the aetiology of neonatal hepatitis and biliary atresia. Arch Dis Child 1977;52:360-7.

4. Ballisteri WF, Bove KE, Ryckman FC. Biliary Atresi \& other Disorders of the Extrahepatic Bile Ducts. In: Suchy FJ, Sokol RJ, Ballisteri WF, editors. Liver Disease in Children. Philadelphia: Lipincott Williams \& Wilkins; 2001. p. 253-174.

5. Bate MD, Bucuvals JC, Alonso MH. Biliary Atresia: Pathogenesis and Treatment. Semin Liver Disease. 1998;18:281-293.
6. Campbell KM, Bezerra JA. Biliary Atresia. In: Walker WA, Kleinman RE, Sherman PM, Shneider BL, Sanderson IR, editors. Pediatric Gastrointestinal Disease. $4^{\text {th }}$ ed. New York: Kluwer Academic Publishers; 2003. p. 1122 - 1123.

7. Davenport M, Savage M, Mowat AP, Howard ER. Biliary atresia splenic malformation syndrome: an etiologic and prognostic subgroup. Surgery 1993; 113:662-8.

8. Carmi R, Magee CA, Neill CA, Karrer FM. Extrahepatic biliary atresia and associated anomalies: etiologic heterogeneity suggested by distinctive patterns of associations. Am J Med Genet 1993;45:683-93.

9. Zukotynski K, Babyn PS. Biliary Atresia [Online]. 2006 [cited 2006 Jun 09]; [4 screens]. Available from: URL: http:// emedicine.medscape.com/article/406335-overview

10. Karpen SJ. Update on the etiologies and management of neonatal cholestasis. ClinicalPerinatology 2002; 29(1): $159-80$.

11. Karim ASMB and Kamal M. Cholestatic jaundice during infancy: Experience in a tertiary care hospital in Bangladesh, Indian Journal of Gastroenterology. 2005; 24:52-54.

12. Sokol RJ, Mark C, Markewicz MR and Karrer FM. Pathogenesis and outcome of biliary atresia: Current concept, Pediatric Gastroentero and Nutri, 2003; 37: 4-21.

13. Kader HHA and Ballisteri WF. Cholestasis. In: Befrman RE, Kleigman RE and Jenson HB, editors. Nelson textbook of pediatrics, $17^{\text {th }}$ edn. Saunders-Elsevier: Philadelphia, 2004: 1668-75.

14. Mandana R, Masoud N and Nazarpour S. Diagnostic value of ultrasonography in biliary duct atresia, Medical journal of Tabriz university of medical sciences, 2009; 31: 31-35.

15. Ikeda S, Sera Y and Akagi M. Serial ultrasonic examination to differentiate biliary atresia from neonatal hepatitis, European journal of pediatrics, 1989; 148: 400.

16. Perk WH. A new diagnostic approach to biliary atresia with emphasis on ultrasonographic triangular cord sign: Comparison of ultrasonography, hepatobiliary scintigraphy and liver needle biopsy in the evaluation of infantile cholestasis, Journal of Pediatric surgery, 1997; 32: 1555-59. 


\section{Table-II}

\section{Age distribution of studied patients at admission $(n=40)$}

\begin{tabular}{|c|c|c|c|c|}
\hline Diagnosis & No & Mean age (days) & Standard deviation & p-value \\
\hline Biliary atresia & 17 & 113.7 & \pm 15.38 & 0.12 \\
\hline NH \& INH & 18 & 105.05 & \pm 16.81 & 0.12 \\
\hline Miscellaneous & 05 & 106.6 & \pm 20.4 & \\
\hline Total patients & 40 & 111.9 & \pm 21.14 & \\
\hline
\end{tabular}

Table-III

Sex distribution of studied patients $(n=40)$

\begin{tabular}{lcccc} 
Sex & Biliary Artesia & INH and NH & Miscellaneous & Total \\
Male & $12(70.6 \%)$ & $10(55.5 \%)$ & $03(60 \%)$ & $25(100 \%)$ \\
Female & $05(29.4 \%)$ & $08(44.4 \%)$ & $02(40 \%)$ & $15(100 \%)$ \\
\hline Total & 17 & 18 & 05 & 40 \\
\hline
\end{tabular}

Table-IV

Age at onset of symptoms and age at admission.

\begin{tabular}{|c|c|c|c|}
\hline \multicolumn{4}{|c|}{ Age at onset of symptoms and age at admission. } \\
\hline & $\begin{array}{c}\text { Biliary atresia }(n=17) \\
\text { Mean } \pm \text { SD }\end{array}$ & $\begin{array}{c}\text { NH \& INH }(n=18) \\
\text { Mean } \pm \text { SD }\end{array}$ & p value \\
\hline Age at onset (days) & $10.1 \pm 4.18$ & $12.4 \pm 4.7$ & 0.73 \\
\hline Age at admission (days) & $113.7 \pm 15.38$ & $105.05 \pm 16.81$ & 0.58 \\
\hline Delay ( days) & 103.6 & 92.65 & \\
\hline
\end{tabular}

Table-V

\begin{tabular}{lcc} 
& \multicolumn{2}{c}{ Clinical features of studied cases. } \\
Clinical characteristics & Biliary & NH/ INH \\
& atresia(n=17) & $(\mathrm{n}=18)$ \\
& No. $(\%)$ & No. $(\%)$ \\
Jaundice & $17(100)$ & $18(100)$ \\
Dark urine & $17(100)$ & $18(100)$ \\
Persistent acholic stool & $15(88.2)$ & $04(22.2)$ \\
Intermittent acholic stool & $02(11.8)$ & $14(77.7)$ \\
Hepatomegaly & $15((88.2)$ & $15(83.3)$ \\
Splenomegaly & $11(64.7)$ & $14(77.7)$ \\
Ascites & $01(5.8)$ & $03(16.6)$ \\
\hline
\end{tabular}

$\mathrm{NH}=$ Neonatal hepatitis

INH = Idiopathic neonatal hepatitis 


\section{Table-VI}

\section{Biochemical parameters of studied cases.}

\begin{tabular}{lccc} 
Liver function tests & $\begin{array}{c}\text { Biliary atresia(n=17) } \\
\text { Mean } \pm \text { SD }\end{array}$ & $\begin{array}{c}\text { NH\& INH(n=18) } \\
\text { Mean+ SD }\end{array}$ & p-value \\
\hline Serum total bilirubin (mg/dl) & $12.4 \pm 2.55$ & $14.7 \pm 4.64$ & 0.04 \\
Serum direct bilirubin (mg/dl) & $7.2 \pm 1.6$ & $11.0 \pm 4.0$ & $<0.01$ \\
Serum albumin (gm/dl) & $32.4 \pm 3.1$ & $30.1 \pm 2.9$ & 0.69 \\
ALT(U/L) & $219.7 \pm 102.5$ & $423.9 \pm 92.6$ & 0.99 \\
Alkaline phosphatase (U/L) & $1048.8 \pm 162.8$ & $629.6 \pm 160.8$ & 0.84 \\
Gamma glutamyl transpeptidase (ãGT) (U/L) & $624.9 \pm 129.5$ & $553.0 \pm 111.2$ & 0.52 \\
Prothrombin time (sec) of patient & $15 \pm 0.49$ & $18.2 \pm 3.9$ & 0.11 \\
INR & $1.44 \pm 2.7$ & $1.5 \pm 0.43$ & 0.28 \\
\hline
\end{tabular}

\section{Table-VII}

Findings of Ultrasonography in studied patients $(n=40)$

\begin{tabular}{lll} 
Ultrasonography findings & Diagnosis & No \\
\hline Small/absent gallbladder with non- & Biliary atresia & 17 \\
visualized biliary channel & INH / NH \& Miscellaneous & 20 \\
Normal Gallbladder with visualized & Cystic lesions were seen in & 03 \\
biliary channel & biliary tree & \\
Choledochal cyst & & \\
\hline
\end{tabular}

\section{Table-VIII}

Liver biopsy findings in studied patients $(n=26)$

\begin{tabular}{llc} 
Findings & Diagnosis & No. \\
\hline Ductular proliferation, bile plugs, & Biliary atresia & 12 \\
intraportal fibrosis & Idiopathic neonatal hepatitis & 10 \\
Inflammation, hepatocyte & Biliary atresia with biliary & 04 \\
necrosis and giant cell & cirrhosis & \\
transformation & & \\
$\begin{array}{l}\text { Ductular proliferation, bile plugs, } \\
\text { inflammation, fibrosis }\end{array}$ & & \\
\hline
\end{tabular}

\section{Discussion:}

This hospital based cross sectional study was carried out to determine the frequency of biliary atresia in infants admitted with cholestatic jaundice along with their clinical profile. During the study period a total of 40 infants were admitted in the Pediatric
Gastroenterology and nutrition department of Bangabandhu Sheikh Mujib Medical University, Dhaka. In the present series of cholestatic jaundice, biliary atresia was found in 17 (42.5\%) cases, neonatal hepatitis in 8 (20\%) and idiopathic neonatal hepatitis in $10(25 \%)$ cases. 
A retrospective study was conducted among Bangladeshi infant to find out the etiology and clinical profile of neonatal cholestatic disorders ${ }^{11}$. A total of 62 infants with cholestatic jaundice were studied who developed jaundice before three months of age and persisted for more than two weeks. In that study biliary atresia was found in 16 (25.8\%), neonatal hepatitis in 22 (35.5\%) and idiopathic neonatal hepatitis in 15 (24.2\%) cases. Neonatal hepatitis was the commonest cause of cholestatic jaundice in that study but biliary atresia was found to be the commonest cause in the present study. This difference may be due to small sample size.

The mean age at admission to hospital of biliary atresia cases was $113.7 \pm 15.38$ days, though the mean age of onset of jaundice was $10.1 \pm 4.18$ days and average delay was 103.6 days. Karim \& $\mathrm{Kamal}^{11}$ reported the mean age at presentation to hospital in their series was 105 days while the mean age at onset of jaundice was 5.8 days and the average delay was 99.2 days. These findings are almost consistent with findings of present study. Delay in diagnosis of cholestatic disorders especially biliary atresia is also a problem in developed countries $^{12}$. If the treatment of extra hepatic biliary atresia is delayed beyond the first 60 days of life, the only option left thereafter is liver transplantation, which is not commonly feasible on a large scale in developing countries. This delay contributes to increase in morbidity and mortality and also to poor outcome ${ }^{13}$.

Amongst the clinical feature only acholic stool was differentiating (88.2\% BA vs. 22.2\% NH). This was also represented by Karim \& $\mathrm{Kamal}^{11}$. There was not much differentiating point as regard to other clinical features. Intermittent acholic stool found in biliary atresia may be due to the progressive obliterative cholangiopathy i.e. incomplete obliteration of entire extrahepatic biliary tree. Conversely in long standing cases of NH/INH persistent acholic stool usually found.

Common signs of the studied cases of biliary atresia were hepatomegaly (88.2\%), splenomegaly (64.7\%) and ascites (5.8\%). In NH/INH cases hepatomegaly was found in $83.3 \%$ cases, splenomegaly in $77.7 \%$ cases and ascites in $6.6 \%$ cases.

Amongst the laboratory findings there were no such differentiating characteristics. However ALT and ALP was found to be raised in NH/ INH and BA respectively but they were not significant statistically.
In biliary atresia cases the mean serum total bilirubin was found to be $12.4 \pm 2.55 \mathrm{mg} / \mathrm{dl}$ and that in NH/INH cases $14.7 \pm 4.64 \mathrm{mg} / \mathrm{dl}$, this value is statistically significant. Serum ALT was found 219.7 $\pm 102.5 \mathrm{U} / \mathrm{L}$ and 423.9 $\pm 92.6 \mathrm{U} / \mathrm{L}$ in biliary atresia and NH/INH cases respectively which is not statistically significant. The mean serum alkaline phosphatase was $1048.8 \pm 162.8 \mathrm{U} /$ $\mathrm{L}$ in biliary atresia and 629.6 $\pm 160.8 \mathrm{U} / \mathrm{L}$ in NH/INH cases. Gamma glutamyl transpeptidase (ãGT), prothrombin time or serum albumin level showed no significant difference between biliary atresia and $\mathrm{NH} /$ INH cases.

The sensitivity and specificity of ultrasonography were $87.5 \%$ and $97.7 \%$ respectively ${ }^{14}$. Ultrasonography was done in all infants with cholestatic jaundice. In biliary atresia cases gallbladder was found either small in size or absent or no contraction of gall bladder was seen even after meals. These findings were consistent with the findings of other studies ${ }^{2}$. Visualization of a normal gallbladder while fasting and contraction after meal virtually rules out biliary atresia cases. But the reverse is not always true ${ }^{15}$. Thus USG of hepatobiliary system both before and after food may be used as differentiating between biliary atresia and $\mathrm{NH} / \mathrm{INH}$.

Though scintigraphy is a competent diagnostic tool, its availability limits its use. Moreover, high jaundice may prevent uptake and further limits its use as a diagnostic tool in the community. The hepatobiliary scintigraphy (HIDA scan) identifies diseased gallbladders and bile drainage problems. Mandana (2009) showed that the sensitivity and specificity of HIDA scan was $100 \%$ and $50 \%$ respectively. Hepatobiliary scintigraphy (using 99 technetium iminodiacetic acids) was done in only selected patients when ultrasonography findings were not consistent with clinical finding. Nine patients showed delayed uptake but normal excretion which is consistent with NH/INH whereas five infants showed normal uptake of the isotope but absent excretion into the biliary channels and intestine which was consistent with biliary atresia. Similar findings were observed by other authors ${ }^{16}$.

Percutaneous liver biopsy is the most valuable procedure in the evaluation of neonatal hepatobiliary diseases and provides the most reliable discriminatory evidence. Biliary atresia is characterized by bile ductular proliferation, the presence of bile plugs and portal and 